\title{
L'évaluation dans le système éducatif en France
}

\section{Jean Claude Emin et Jaqueline Levasseur}

L'article présente les dispositifs d'évaluation conduits en France par la Direction de l'Evaluation, de la Prospective et de la Performance, pour le compte du ministère de l'éducation nationale français. Ces dispositifs nationaux et internationaux s'appuient sur les méthodes de la mesure en éducation et les modèles psychométriques. Ils fournissent les informations permettant aux responsables de la politique éducative d'apprécier les performances et l'efficacité du système éducatif et de prendre des décisions.

Depuis le début des années 1970 les ministres de l'Éducation nationale se sont attachés à renforcer le suivi et l'évaluation du système éducatif par la création d'une direction qui, dans le ministère, est spécifiquement chargée de contribuer à l'évaluation et au pilotage du système éducatif.

Ainsi, la plus grande partie des travaux d'évaluation du système éducatif est réalisée, en France, par la Direction de l'évaluation, de la prospective et de la performance (DEPP) qui est une direction de l'administration centrale du ministère et non, comme dans la plupart des pays, une agence ou un institut lié à une université Ce mode d'organisation mis en place depuis maintenant près de trentecinq ans présente, aux yeux des responsables du système éducatif français, plusieurs avantages. La Direction:

- organise et gère le système d'information sur l'éducation;

- réalise des travaux d'évaluation;

- conçoit des outils de pilotage et les met à la disposition des responsables du système et des enseignants;

- réalise des études sur le système éducatif.

Le fait de disposer pour réaliser ces trois dernières tâches d'un lien direct avec le système d'information - qui s'appuie en France sur une solide tradition de centralisation - est incontestablement un atout important qui facilite certaines réalisations. Par ailleurs, cette Direction constitue une "passerelle» avec les milieux de la recherche en éducation. Au plan national comme au plan international, elle participe au pilotage et à l'animation des recherches conduites dans le domaine éducatif. 
Le fait d'être une structure interne au ministère permet de mieux prendre en compte les besoins des décideurs. Cela facilite, auprès des responsables et des acteurs, la diffusion des résultats de ses travaux et leur prise en compte par les réseaux de formation national, régional du ministère. En outre une telle structure a une mémoire, garantissant la continuité et la cumulativité des travaux que ne permet pas toujours le recours à des institutions externes. L'expérience de ces dernières décennies, montre qu'une telle organisation est parfaitement compatible avec l'indépendance scientifique nécessaire aux travaux d'évaluation, d'autant plus que les résultats de ces travaux sont systématiquement rendus publics.

Les travaux de la DEPP alimentent les décisions politiques; ainsi, les résultats des évaluations nationales et internationales réalisées par la DEPP ont nourri les réflexions qui ont conduit à définir un «socle commun de compétences et de connaissances», ensemble de compétences-clefs que le système éducatif doit s'efforcer de faire acquérir à chaque jeune en fin de scolarité obligatoire, ce qui constitue une évolution majeure pour le système éducatif français.

De plus, les évaluations réalisées par la DEPP ont largement sous-tendus les réflexions et propositions du Haut Conseil à l'évaluation de l'école (HCée), instance indépendante mise en place en 2000 et qui a, pendant cinq ans, évalué l'évaluation.

Ces travaux contribuent aussi pour une part, à impulser la recherche en éducation, tant par le rôle incitatif que la DEPP peut jouer par des appels d'offres en direction des chercheurs que par la base de travail que constituent pour ceux-ci les nombreuses données qu'elle recueille et analyse.

Enfin, ils se sont inscrits, voire ont parfois précédé un mouvement essentiel en France et ailleurs en rendant compte des résultats et de la performance du service public national de l'éducation, contribuant ainsi à conforter une exigence de résultats dans les services publics.

La DEPP développe - à la demande du ministre et de la direction pédagogique du ministère - plusieurs dispositifs, dans un contexte où la volonté de faire jouer à l'évaluation un rôle essentiel dans le pilotage et la régulation des décisions de politique éducative. Les problématiques et les thématiques sont arrêtées en concertation entre les différentes instances de décisions du ministère de l'Éducation national.

\section{Une évaluation du système éducatif dans son ensemble: l'état de l'école}

L'État de l'école, depuis près de quinze ans, remis chaque année aux responsables de la politique éducative, fournit une trentaine d'indicateurs nationaux, assortis de comparaisons internationales. ${ }^{1}$

Le parti retenu depuis l'origine a été:

- de donner une image synthétique de l'ensemble du système éducatif, de 
l'école maternelle à l'enseignement supérieur et jusqu'à la formation continue des adultes. L'état de l'école s'intéresse à la politique éducative et décrit tout le système éducatif, aussi bien public que privé;

- de permettre une appréciation de l'efficacité et de l'efficience du système éducatif: une distinction est faite entre indicateurs de coûts, indicateurs d'activités et indicateurs de résultats du système éducatif. Rapprocher les activités des coûts permet d'apprécier l'efficacité (que fait le système éducatif avec l'argent qui lui est alloué par la société française ?). S’intéresser aux résultats permet de se faire une idée de l'efficience (atteint-on les objectifs fixés et avec quel «rendement»?).

Les indicateurs de résultats s'efforcent de réaliser cette appréciation en se référant aux objectifs que la société française assigne à son école, et que la loi rappelle:

- donner un capital de connaissances et de compétences; ceci se mesure notamment grâce à un large dispositif d'évaluations-bilans et aux comparaisons internationales;

- participer à la formation des futurs citoyens; cet objectif s'apprécie grâce à des enquêtes spécifiques - des enquêtes déclaratives - qui ont été menées à différents niveaux de l'enseignement secondaire;

- préparer à une bonne insertion sur le marché du travail, le troisième objectif fixé par la loi au système éducatif, fait lui aussi l'objet de plusieurs indicateurs, comme par exemple: «le diplôme et le risque de chômage» et «l'emploi et le devenir professionnel des diplômés de l'enseignement supérieur».

Ces trois objectifs sont généralement ceux de tout système éducatif. Mais, nous sommes particulièrement attachés en France à un quatrième objectif: l'équité, objectif qui fait lui aussi l'objet d'indicateurs, comme celui relatif au «niveau de formation atteint selon le milieu social des élèves».

L'intérêt, pour le pilotage d'ensemble du système, des analyses temporelles que permet ce état de l'École, apparaît incontestable. Elles sont au cœur des débats et fondent les orientations actuelles de la politique éducative. ${ }^{2}$ On peut les résumer ainsi en quelques lignes.

Le système éducatif français a connu au cours de la deuxième moitié du XXe siècle un développement important qui s'est traduit par une élévation considérable des niveaux de qualification et de formation: pour en prendre un indicateur significatif, en 2004, parmi les jeunes sortis de formation initiale, 42\% sortaient diplômés de l'enseignement supérieur; vingt ans auparavant, cette proportion n'était que de $15 \%$. Par ailleurs, respectivement $7 \%$ et $10 \%$ des sortants obtenaient en 2004 pour diplôme le plus élevé un certificat d'aptitude et un brevet d'études professionnelles, $6 \%$ des jeunes quittaient le système éducatif sans qualification; ils étaient $27 \%$ il y a 20 ans.

Mais après des décennies de progression, tous les indicateurs montrent de manière frappante le palier que connaît notre système éducatif depuis le milieu des années quatre-vingt dix: l'accès au niveau du baccalauréat ou à l'enseignement supérieur, la proportion d'une génération ayant le baccalauréat etc., ne progres- 
sent plus. Le nombre de sortants du système éducatif sans diplôme ne diminue plus.

Ce constat rend indispensable le développement d'outils d'évaluation qui permettent d'apprécier les résultats du système éducatif au plus près du terrain dans les établissements et dans les classes.

\section{Les dispositifs pour le pilotage pédagogique}

Depuis près de quatre décennies la France met en place - à la demande des responsables de la politique éducative - des dispositifs d'évaluation des acquis des élèves. Ces dispositifs fournissent des informations donnant à tous les niveaux de décisions - local et national - les moyens d'un pilotage pédagogique (Remond, 2005).

Au plan conceptuel, nous distinguons les dispositifs "d'évaluation diagnostique» et les dispositifs "d'évaluation bilan» qui constituent deux types d'évaluation qui ne se substituent pas l'un à l'autre. Ils different dans leurs objectifs, dans les modalités de mise en œuvre, dans l'exploitation et l'utilisation des résultats.

Ces deux types de dispositifs s'intéressent aux connaissances et compétences que les programmes nationaux demandent aux élèves d'acquérir. Les activités scolaires demandent aux élèves de réaliser des tâches complexes qui mettent en interrelation des connaissances, des compétences, des représentations. Évaluer isolément une compétence n'est donc pas facile. La lecture des travaux de recherche montre des approches et des définitions différentes de "compétence». Celle des programmes scolaires témoigne aussi de cette polysémie. C'est alors un sens très global qui est retenu: l'élève est compétent s'il satisfait dans un contexte précis aux exigences attendues de lui dans les programmes scolaires.

\section{Les évaluations diagnostiques}

Les évaluations diagnostiques - dans la conception française - sont des outils professionnels pour les enseignants dans leur classe. Elles concernent toutes les classes, tous les élèves. Elles permettent d'établir un diagnostic individuel - évaluer les points forts et les points faibles (freins aux apprentissages), d'aider l'enseignant à définir les actions pédagogiques adaptées à la situation de chacun et à réguler la programmation des apprentissages.

Les outils de ces évaluations sont:

- Des protocoles d'évaluation en français et en mathématiques, élaborés chaque années avec des représentants des corps d'inspection et des enseignants. Cette évaluation est mise en place depuis 1989, à chaque rentrée scolaire pour les élèves entrant en 3ème année et en Gème année de scolarité obligatoire. Sur le même principe, entre les années 1992 et 2002 des protocoles d'évaluation diagnostique ont été proposés dans toutes les disciplines aux élèves entrants en 10ème année de scolarité; 
- Une banque d'outils d'aide à l'évaluation diagnostique des élèves, couvrant tous les niveaux scolaires et un large ensemble de disciplines, est mise à la disposition des enseignants qui peuvent y recourir à tout moment de l'année scolaire. Ces outils d'évaluations diagnostiques sont avant tout prévus pour une utilisation directe en classe, ce sont eux qui permettent aux enseignants de suivre l'évolution et le degré d'acquisition des compétences de chacun.

\section{Quelles utilisations de ces évaluations?}

Par les enseignants dans la gestion pédagogique de leur classe

Ces évaluations diagnostiques sont des descripteurs des réussites et échecs de chaque élève, des aides à la constitution de groupes de groupes de besoin, des supports pour la réflexion pédagogique. Elles permettent aux enseignants d'identifier:

- les élèves en difficulté pour lesquels une remédiation est indispensable, avec la mise en place de Programmes Personnalisés d'Aide et de Progrès (PPRE);

- les élèves pour lesquels un simple rappel ou un renforcement des acquis est juste nécessaires pour ancrer les nouveaux apprentissages;

- les difficultés de tous les élèves: l'analyse des résultats doit se faire pour tous les élèves et non uniquement pour ceux qui ont des scores moyens ou faibles.

\section{Au niveau national}

Elles fournissent des repères ${ }^{3}$ calculés sur des échantillons représentatifs d'élève, permettant aux enseignants de situer les performances de leurs élèves et de leur classe dans l'ensemble national (Brezillon, Chollet-Remvikos \& Rebmeister, 2003).

Les protocoles étant nouveaux chaque année, les résultats sont valables pour l'année en cours. C'est pourquoi, les résultats nationaux:

- sont des repères;

- sont un constat sans autre ambition que de décrire une situation caractérisée à un moment donné;

- aident les enseignants à faire une analyse individuelle des freins que rencontrent leurs élèves dans les apprentissages;

- ne constituent pas le reflet de l'acquisition du programme officiel;

- ne peuvent - au sens psychométrique - être comparés d'une année à l'autre;

- ne sont pas des indicateurs d'évolution du système éducatif.

Les évaluations bilan

Les évaluations bilan sont des outils pour les décideurs politiques, responsables le pilotage d'ensemble du système éducatif. Leur méthodologie de construction s'appuie sur les méthodes de la mesure en éducation et les modèles psychométriques (Vrignaud, 2001a, 2002, 2003). Elles concernent des échantillons représentatifs d'établissement, de classes et d'élèves. Elles sont organisées en fin de 
cycles. Elles révèlent, en référence aux objectifs de la politique éducative, les objectifs atteints et ceux qui ne le sont pas. Ces évaluations permettent d'agir au niveau national sur les programmes des disciplines, sur les organisations des enseignements, sur les contextes de l'enseignement, sur des populations caractérisées.

La finalité, les objectifs et la méthodologie de construction des évaluations bilans ne permettent pas de rendre compte de résultats individuels pertinents pour chaque élève ou chaque classe ou chaque établissement. Par contre, le recueil des informations sur des échantillons représentatifs conduit à un bilan global national ou régional des acquis des élèves, à en étudier l'évolution. Elles répondent à un but précis: confronter, sur la base d'un constat objectif, les résultats du fonctionnement pédagogique du système éducatif aux objectifs nationaux qui lui sont assignés à des étapes importantes du cursus scolaire. Outils d'aide à la décision pour la politique éducative, elles fournissent les indicateurs de pilotage local et national du système éducatif par des résultats pédagogiques.

Fortement développées de la fin des années 70 à la fin des années 80, elles ont été peu à peu délaissées (Salines \& Vrignaud, 2001). Depuis les années 2000, elles retrouvent leur place dans la politique d'évaluation du système éducatif. Le cycle d'évaluation de 2003 à 2008 permet de rassembler et d'analyser un capital d'informations sur les connaissances et les compétences des élèves en fin d'école primaire (cinq années de scolarité) et en fin de collège (quatre années de scolarité). La reprise d'un cycle identique en 2009 permettra de fournir aux responsables du système éducatif des comparaisons dans le temps. La succession des champs disciplinaires dans l'organisation du cycle a été arrêtée en concertation en respectant les priorités de la politique du ministre. Le cycle ${ }^{4}$ actuel couvre:

année 2003, évaluation en fin d'école des compétences dans la maîtrise des langages et de la langue française; en fin de collège des compétences générales; année 2004, évaluation des connaissances et des compétences dans la pratique d'une langue vivante étrangère, allemand et anglais en fin d'école, allemand, anglais et espagnol en fin de collège;

année 2005, évaluation des attitudes des écoliers et des collégiens à l'égard de la vie en société;

année 2006, évaluation des connaissances et des compétences en histoire, géographie, éducation civique;

année 2007, évaluation des connaissances et des compétences en sciences expérimentales (physique, chimie, sciences de la vie et de la terre);

année 2008 évaluation des connaissances et compétences en mathématiques; année 2009, reprise du cycle.

Chaque année la collecte comprend une prise d'informations sur le contexte dans lequel se déroulent les enseignements à partir de questionnaires proposés aux élèves, aux enseignants, aux directeurs d'école, aux chefs d'établissements.

Les performances observées sont décrites dans des échelles de compétences, pour chaque échelle, six groupes d'élèves correspondant à des niveaux différen- 
ciés de maîtrise de la compétence sont distingués (Beuzon, Dos Santos \& Trosseille 2005; Dos Santos, Gibert \& Yacoub, 2006; Gibert, Levasseur \& Pastor, 2004). Les échelles de référence ainsi constituées permettront de voir comment la distribution des élèves dans les différents niveaux de performance évolue dans le temps, lors des reprises successives de l'évaluation à chaque cycle.

\section{Quelles informations pour les responsables de la politique éducative?}

L'édition et la diffusion des résultats concernent en premier les responsables et décideurs du système éducatif au niveau régional et national. Ces résultats permettent entre autres de:

- de connaître les facteurs malléables sur lesquels le système éducatif pourrait directement agir (objectifs, contenus, structures) pour améliorer la réussite des élèves;

- suivre l'évolution dans le temps des changements, et innovations demandés au système éducatif;

- de comparer des populations, des structures, des groupes caractérisés;

- de mettre en relation les évolutions des acquis des élèves et les effets dus aux évolutions des contextes de l'enseignement.

Par ailleurs, les échelles conduisent à s' interroger sur la question du seuil à partir duquel les objectifs du programme sont atteints.

La construction de l'échelle donne la répartition des réussites des élèves. L'échelle ne constitue pas une norme au-delà de laquelle les exigences du programme sont atteintes et en deçà ne le sont pas, elle constate l'hétérogénéité des élèves dans la maîtrise des compétences attendues en fin d'école et en fin de collège.

La question des compétences devant être considérées comme nécessaires en fin de collège, soit dans un objectif de poursuite d'études, de quelque type qu'elles puissent être, soit pour s'insérer harmonieusement dans la vie professionnelle et sociale.

Le bilan collège vient confirmer ce qui était perceptible dans les analyses des évaluations internationales à savoir que les élèves sont plus à l'aise dans le prélèvement d'information (compétence considérée comme de bas niveau) alors qu'ils éprouvent des difficultés dans l'exploitation complexe de l'information contenue dans un document (compétence considérée comme de haut niveau). Ceci ouvre la réflexion sur les points à faire travailler et approfondir par les élèves au collège.

En outre, les analyses font ressortir des différences entre filles et garçons, des disparités entre groupes d'élèves à la fin de l'école primaire et à la fin du collège, des disparités des résultats qui restent marquées au fil des années entre les $10 \%$ des élèves les plus forts et les $10 \%$ des élèves les plus faibles, des résultats des élèves en ZEP inférieurs à ceux des élèves qui ne sont pas en ZEP, des disparités géographiques.

La grande hétérogénéité des élèves sortant du collège implique une réflexion sur la prise en compte des difficultés de ces élèves dès l'entrée en Gème, puisque 
l'on sait qu'elles sont déjà constituées à ce moment et ne feront que s'amplifier dans la mesure où la construction des compétences de plus haut niveau s'étaye sur celles du niveau le plus bas ainsi qu'une réflexion sur la prise en compte des difficultés de ces élèves à l'entrée en seconde, qu'elle soit professionnelle ou générale et technologique.

L'ensemble des informations disponibles donne des éléments de pilotage et de régulation de la politique et a contribué notamment à décider de politiques telles que la définition d'un réseau «ambition-réussite» pour les écoles et les collèges les plus en difficultés, la définition de l'acquisition d'un socle de connaissances et compétences exigibles pour tous les élèves à la fin de leur scolarité obligatoire.

\section{Les évaluations pour les comparaisons internationales}

Les évaluations internationales auxquelles participe la France (Bottani \& Vrignaud, 2005), notamment PISA - évaluation des compétences des élèves de 15 ans réalisée sous l'égide de l'OCDE -, PIRLS - évaluation des élèves de 9 ans réalisée dans le cadre de l'IEA ou des évaluations conduites avec des partenaires européens tel que l'élaboration d'une banque d'items européenne pour évaluer les compétences en langues vivantes étrangères (EBAFLS), sont autant d'informations qui complètent et enrichissent la réflexion nationale (Cytermann \& Demeuse, 2005).

\section{Que nous apprennent les comparaisons internationales L'exemple du programme PISA}

Les informations rassemblées dans le cadre du programme PISA permettent, au-delà de comparaisons aboutissant à un palmarès des pays, d'étudier les résultats en analysant les facteurs de contexte associés à la performance des élèves (Bourny, Dupé, Rémond, Robin \& Rocher, 2002; Bourny, Fumel, Monnier \& Rocher, 2004).

Le but premier du PISA consiste à comparer les acquis des jeunes de 15 ans des pays participants en tenant compte des spécificités propres à chaque système éducatif. Les indicateurs comparables au plan international permettent d'établir les niveaux de compétences atteints dans les différents pays (Rocher, 2002).

Or, ce sont les indicateurs de base, ceux qui décrivent hors contexte la maîtrise des compétences des élèves de 15 ans qui ont été le plus exploités par les médias en ne retenant que les aspects de palmarès, alors que les écarts des performances entre pays peuvent être étudiés bien sûr au regard des différentes variables contextuelles liées à l'élève, à son milieu socio-culturel, à l'établissement, mais aussi aux choix de politiques éducatives propres à chaque pays (Duru-Bellat, Mons \& Suchaut, 2004).

Ainsi, en $2000^{5}$, sur l'ensemble de l'épreuve de compréhension de textes du PISA, les élèves de France réalisent des performances qui se situent dans la 
moyenne des pays de l'OCDE. Mais en fait, les élèves français de 15 ans se répartissent en deux pôles aux résultats très contrastés: ceux qui sont au lycée (10éme année de scolarité) et ont donc eu une scolarité sans redoublement (près de la moitié des élèves de 15 ans) ont des performances moyennes qui les situent au dessus des pays les plus performants à l'enquête. Ceux qui sont encore au collège, ont donc redoublé une fois (plus du tiers des élèves de 15 ans) ont, au contraire, des résultats qui les situent au niveau moyen des pays les moins bien classés.

Il faut, par ailleurs, souligner que les élèves de 15 ans considérés comme étant en grande difficulté de lecture sont proportionnellement moins nombreux que dans la moyenne des autres pays: ils représentent $4,2 \%$ de la population contre $6,2 \%$ sur l'ensemble des pays de l'OCDE. Cette approche comparative permet donc de relativiser la proportion d'élèves et de jeunes en difficulté telle qu'elle ressort des enquêtes menées en France.

En outre, en France, le décret de juillet 2006 en application de la loi sur l'éducation, relatif au socle commun de connaissances et de compétences, fait explicitement référence au programme PISA.

\section{Un outil d'evaluation d'établissement scolaire: les indicateurs de performance des lycées}

En France, l'évaluation des établissements est une préoccupation relativement récente. Plusieurs raisons peuvent sans doute l'expliquer. Tout d'abord, on sait qu'une forte tradition de centralisation a longtemps marqué l'école française. Dans une telle tradition, il était logique de penser a priori que les différences de fonctionnement d'une école à l'autre sont faibles et que l'école n'est pas un niveau d'analyse pertinent. Ensuite, des considérations idéologiques allaient généralement de pair avec cette tradition de centralisation. On peut les résumer rapidement ainsi: il est nécessaire et juste que tous les établissements fonctionnent de la même façon et - si l'on caricature quelque peu - les différences de fonctionnement et de performances entre eux sont condamnables. Enfin, certains considèrent que la sociologie de l'éducation, dont les travaux sont très développés en France, a joué un rôle de frein en la matière. En mettant en avant l'appartenance sociale comme facteur déterminant de la réussite scolaire, elle reléguait à l'arrière plan le fait que l'école pouvait faire la différence.

La situation a évolué au cours des vingt à vingt-cinq dernières années et l'idée que tous les lycées peuvent être différents - ce qui est légitime - mais qu'ils n'offrent pas toujours la même qualité de service - ce qui l'est moins - est maintenant admise.

C'est d'autant plus le cas que les lycées français sont, depuis une vingtaine d'années, dotés d'une large autonomie dans le cadre de la décentralisation du système éducatif. Ils mettent en oeuvre une politique éducative qui reste nationale dans sa définition, mais doivent élaborer un projet particulier qui définit les mo- 
dalités de mise en oeuvre des objectifs et des programmes nationaux dans la situation concrète qui est la leur.

Par ailleurs, comme on l'a évoqué plus haut, une demande sociale de plus en plus forte en matière d'informations sur les résultats et surtout sur l'efficacité des établissements a accompagné la prise de conscience par l'ensemble de la société française du rôle de l'école dans la réussite.

La presse s'est employée très tôt à satisfaire cette demande en publiant des palmarès de lycées établis en fonction du taux de réussite de leurs élèves au baccalauréat, "palmarès» qui étaient directement inspirés de la logique du ranking à l'anglo-saxonne. Ces "palmarès» ont immédiatement fait l'objet de deux critiques fortes de la part des enseignants et des responsables d'établissement.

- S'intéresser au seul taux de réussite au baccalauréat, c'est accepter de juger un établissement uniquement sur le «dernier obstacle de la course» que constitue la scolarité dans un lycée. C'est négliger le fait que le lycée peut sélectionner ses élèves pour ne conserver «en fin de course» que ceux dont il juge qu'ils vont réussir le baccalauréat;

- S'intéresser au seul taux effectif de réussite au baccalauréat, c'est laisser de côté l'action propre du lycée, ce qu'il a «ajouté», voire «retranché» au niveau initial des élèves qu'il a accueillis. En d'autres termes, si un lycée a un bon taux de réussite au baccalauréat, est-ce dû au fait qu'il a reçu (voire sélectionné) de bons élèves, dotés de bonnes méthodes de travail, qui ont pu obtenir le baccalauréat sans effort particulier de sa part, ou bien, est-ce dû au fait qu'il a su, tout au long d'une scolarité, développer chez des élèves peut-être moins bien dotés au départ, les connaissances et les capacités qui ont permis leur succès?

C'est pourquoi les indicateurs de performance, rendus publics depuis maintenant près de $10 \mathrm{ans}^{6}$, combinent deux principes qui visent à répondre directement aux deux critiques faites aux «palmarès de lycées» en proposant, d'une part, de porter plusieurs regards sur la performance d'un lycée, et en s'efforçant, d'autre part, d'apprécier la «valeur ajoutée» de ce lycée.

Trois indicateurs sont proposés, tous construits en fonction de l'objectif essentiel qui est fixé aux lycées:

- Le taux de réussite au baccalauréat; c'est l'indicateur le plus traditionnel, le plus connu et surtout le plus facile à établir. Il rapporte le nombre d'élèves du lycée reçus au baccalauréat au nombre d'élèves présentés. Mais on sait qu'il est insuffisant pour rendre compte de l'efficacité d'ensemble d'un lycée;

- Le taux d'accès au baccalauréat; cet indicateur évalue, pour un élève entrant dans un lycée la probabilité qu'il obtienne le baccalauréat à l'issue d'une scolarité entièrement effectuée dans le lycée, et ceci quel que soit le nombre d'années nécessaire. Contrairement au précédent, cet indicateur s'intéresse à l'ensemble de la scolarité dans le lycée;

- La proportion de bacheliers parmi les sortants présente, parmi les élèves qui ont quitté l'établissement, quelles qu'en soient les raisons, la proportion de 
ceux qui l'ont quitté avec le baccalauréat. Un lycée qui sélectionne ses élèves et qui n'autorise pas (ou très peu) le redoublement aura, pour cet indicateur, une valeur plus faible qu'un lycée qui laisse plusieurs chances à ses élèves.

Pour chacun de ces indicateurs, une appréciation de la «valeur ajoutée» de l'établissement, c'est-à-dire de son efficacité propre, est calculée en tenant compte des caractéristiques des élèves qu'il accueille.

\section{Conclusions}

L'ensemble de ce système d'évaluation, composé de dispositifs divers et complémentaires les uns des autres, s'efforce de présenter des résultats aux responsables des prises décisions à tous niveaux.

Trois points méritent d'être soulignés:

- C'est un système dynamique qui a pour ambition de "couvrir» progressivement tous les aspects du système éducatif et doit constituer un ensemble cohérent, permettant d'apprécier les résultats et de fournir des outils de pilotage aussi bien au niveau national - pour le ministre et le Parlement - qu'au niveau de la classe - pour chaque enseignant;

- Ce système est l'objet d'un effort très important de diffusion publique, de formation et d'information. La transparence des résultats de ces travaux est une exigence de démocratie et d'efficacité. Il est essentiel de donner à tous ceux qui doivent s'en servir, les moyens de le faire au mieux et de façon efficace. Les données publiques et largement diffusées sont une source importante pour les chercheurs en science de l'éducation;

- Enfin, il faut souligner une idée essentielle d'un point de vue déontologique: Ces travaux d'évaluation permettent d'éclairer les décisions des responsables. Ils les aident à formuler des hypothèses d'actions et ils leur permettent de vérifier le bien fondé de ces hypothèses. Ils constituent ainsi des outils indispensables à un exercice éclairé de leurs responsabilités politiques, mais ils ne donnent pas de solutions toutes faites aux problèmes rencontrés dans le système éducatif.

\section{Notes}

1 On peut consulter l'état de l'école, comme l'ensemble des travaux et publication de la Direction de la programmation et du développement sur le site du ministère de l'Éducation nationale: http://www.education.gouv.fr

2 Largement diffusé (45 000 exemplaires) l'état de l'École est une référence. Ses indicateurs sont souvent utilisés par les parlementaires lors des débats sur l'éducation, en particulier au moment de la discussion du budget; les journalistes y ont également recours.

3 Notes d'évaluation «les réponses des élèves de CE2 à l'évaluation de septembre 2003" $\mathrm{n}^{\circ}$ 04-05 mai 2004, «l'évaluation des compétences des élèves de sisième en septembre 2003» nº4-06 mai 2004. 
4 Notes d'évaluations $n^{\circ}$ 04-09 et 04-10 octobre 2004; $\mathrm{n}^{\circ}$ 05-0-, 05-07, 05-08, 05-09, 0510 , septembre 2005; n 06-02 août 2006.

5 Notes d'évaluation «les élèves de 15 ans premiers résultats d'une évaluation internationale des acquis des élèves (PISA») $n^{\circ}$ 01-52 décembre 2001.

6 Ces indicateurs peuvent être consultés sur le site Web du ministère: http://www.education.gouv.fr

\section{Bibliographie}

Beuzon, S., Dos Santos S. \& Trosseille, B. (2005). Les compétences en anglais des élèves en fin de collège. Note d'évaluation DEPP, 05-08.

Bottani, N. \& Virgnaud, P. (2005). La France et les évaluations internationales. Rapport établi à la demande du Haut Conseil de l'Évaluation de l'École, 16, 180.

Bourny, G., Dupé, C., Rémond, M., Robin, I. \& Rocher, T. (2002). Les compétences des élèves français à l'épreuve d'une évaluation internationale - Premiers résultats de l'enquête PISA 2000. Les Dossiers de la DP\&D, 137.

Bourny, G., Fumel, S. Monnier, A.-L. \& Rocher, T. (2004). Les élèves de 15 ans - Premiers résultats de l'évaluation internationale PISA 2003. Note d'évaluation DEP, 04.12.

Brezillon, G., Chollet-Remvikos, P. \& Rebmeister, B. (2003) . Évaluations CE2-sixième-cinquième: repères nationaux septembre 2002. Les Dossiers de la DEP, 141, 433.

Ceard, M.-R., Remond, M. \& Varier, M. (2003). L'appréciation des compétences des élèves et des jeunes en lecture et en écriture et l'évolution de ces compétences dans le temps. Rapport établis à la demande du Haut Conseil de l'Évaluation de l'École, 11, 147.

Cytermann, J.-R. \& Demeuse, M. (2005). La lecture des indicateurs internationaux en France. Rapport établi à la demande du Haut Conseil de l'Évaluation de l'École.

Dauphin, L., Rebmeister, B. \& Zelty, C. (2003). L'évaluation des compétences des élèves de cinquième en septembre 2002. Note d'information - DPD.

Dos Santos, S., Gibert, F. \& Yacoub, S. (2006). Les attitudes à l'égard de la vie en société des élèves de fin d'école et de fin de collège. Note d'évaluation, 06-06.

Duru-Bellat, M., Mons, N. \& Suchaut, B. (2004). Inégalités sociales entre élèves et organisation des systèmes éducatifs - quelques enseignements de l'enquête PISA. Les notes de l'IREDU, 04/02.

Forestier, C., Thelot, C. \& Emin, J. C. (2007). Que vaut l'enseignement en France. Paris: Editions Stock.

Gibert, F., Levasseur, J. \& Pastor, J.-M. (2004). La maîtrise du langage et de la langue française en fin d'école primaire. Note d'évaluation DEPP, 04-10.

Levasseur, J. (2006). Le programme international pour le suivi des acquis des élèves (PISA). Revue internationale d'éducation SEVRES, 43, 127-140.

Murat, F. \& Rocher, T. (2002-2003). La place du projet professionnel dans les inégalités de réussite scolaire à 15 ans. France Portrait Social, INSEE.

Poujeau, J. (2003). Une sociologie d'Etat: l'école et ses experts en France. Paris: Editions Raison d'agir.

Robin, I. \& Rocher, T. (2003). La compétence en lecture des jeunes de 15 ans: une comparaison internationale. Données Sociales, INSEE.

Rocher, T.( 2003). La méthodologie des évaluations internationales de compétences. Psychologie et Psychométrie, 2/3, 117-146.

Remond, M. (2005). Regards croisés sur les évaluations institutionnelles. Repères, 31, 113-140.

Salines, M. \& Vrignaud, P. (2001). Apprécier et certifier les acquis des élèves en fin de collège: diplôme et évaluations-bilans. Rapport établi à la demande du Haut Conseil de l'Évaluation de l'École, 2. 
Vrignaud, P. (2001a). Le fonctionnement différentiel des items: méthodologie du contrôle des biais dans l'adaptation des épreuves pour les enquêtes internationales. In C. Sabatier \& P. Dasen (Eds), Cultures, développements et éducation. Autres enfants, autres écoles (pp. 185197). Paris: L'Harmattan.

Vrignaud, P. (2001). Évaluations sans frontières: comparaisons interculturelles dans le domaine de la cognition. In M. Huteau (Ed.), Les figures de l'intelligence (pp. 79-115). Paris: Éditions et Applications Psychologiques.

Vrignaud, P. (2002). Les biais de mesure: savoir les identifier pour y remédier. Bulletin de Psychologie, 55, (6), 625-634.

Vringaud, P. (2003). Objectivité et authenticité dans l'évaluation. Avantages et inconvénients des Questions à Choix Multiples et des Questions à Réponses Complexes: importance du format de réponse pour l'évaluation des compétences verbales. Psychologie et Psychométrie, 24, 147-188.

Etat de l'Ecole - de la maternelle à l'université (2006). 30 indicateurs sur le système éducatif français. Publication de la DEPP, 16.

Dix-huit questions sur le système éducatif: synthèses de travaux de la DEP (2003). Éducation et formations, 66, spécial.

Repères - Références statistiques année 2006, publication DEPP.

\section{Si toghraphie:}

http://www.educ-eval.education.fr/

http://cisad.adc.education.fr/hcee/documents/

Mots-clés: Évaluation des systèmes éducatifs, méthodes de mesures en éducation, pilotage du système éducatif, efficacité du système éducatif

\section{Evaluation im französischen Bildungssystem}

\section{Zusammenfassung}

Der Artikel behandelt die Bestimmungen zur Leistungsbewertung, die im Auftrag vom französischen Ministerium für Erziehung von der DEP (Direction de l'Evaluation, de la Prospective et de la Performance) in Frankreich festgelegt wurden. Diese nationalen und internationalen Bestimmungen stützen sich auf die Bewertungsmethoden auf dem Gebiet der Erziehung sowie auf die psychometrischen Modelle. Sie liefern Informationen, die es den Verantwortlichen der Schulpolitik erlauben, die Leistungen und die Wirksamkeit des Erziehungssystems zu bewerten sowie die entsprechenden Entscheidungen zu treffen.

Schlagwörter: Evaluation des Bildungssystems, Messmethoden, Steuerung des Bildungssystems, Effektivität des Bildungssystems 


\section{La valutazione nel sistema educativo in Francia}

\section{Riassunto}

L'articolo verte sui dispositivi di valutazione adottati in Francia dal DEP (Direction de l'Evaluation, de la Prospective et de la Performance) per conto del Ministero dell'Educazione Nazionale Francese. Questi dispositivi nazionali e internazionali si basano sui metodi di valutazione in campo educativo e sui modelli psicometrici. Essi forniscono le informazioni che consentono ai responsabili della politica scolastica di valutare le prestazioni e l'efficacia del sistema educativo, come pure di prendere le decisioni del caso.

Parole chiave: Valutazione dei sistemi educativi, metodi di misura in educazione, politica educativa, efficacia del sistema educativo

\section{Assessment in the French Educational System}

\section{Summary}

The article concerns the assessment systems adopted in France by DEP (Direction de l'Evaluation, de la Prospective et de la Performance) on behalf of the French Ministry of National Education. These national and international systems are based on methods of assessment in the field of education and on psychometric models. They provide the persons in charge of educational policy with information, thus allowing them to assess the performances and the effectiveness of the educational system, as well as to take the necessary decisions.

Key-words: Evaluation of educational systems, methods of educational assessment, educational policy, effectiveness of eduactional system 